\title{
INTRATHORACIC TRACHEAL RECONSTRUCTION WITH A COLLAGEN-CONJUGATED PROSTHESIS: EVALUATION OF THE EFFICACY OF OMENTAL WRAPPING
}

Masayoshi Teramachi, MD ${ }^{a}$

Norihito Okumura, $\mathrm{MD}^{\mathrm{a}}$

Tatsuo Nakamura, $\mathrm{MD}^{\mathrm{a}}$

Yasumichi Yamamoto, $\mathrm{MD}^{\mathrm{a}}$

Tetsuya Kiyotani, MD ${ }^{a}$

Yukinobu Takimoto, $\mathrm{MD}^{\mathrm{a}}$

Shojiro Matsuda, $\mathrm{MAg}^{\mathrm{b}}$

Yoshito Ikada, PhD ${ }^{b}$

Yasuhiko Shimizu, MD ${ }^{a}$
Reconstructions of the intrathoracic trachea in 24 dogs were done with the use of $50 \mathrm{~mm}$ long collagen-conjugated tracheal prostheses. Omental wrapping was also done in 14 of the dogs (omentopexy group) to evaluate the efficacy of this option in comparison with results in the other 10 dogs (control group). All 24 dogs had uneventful postoperative courses and were killed at 4 weeks or 3, 6, or 12 months after the operation. Better epithelialization and fewer complications, such as mesh exposure and luminal stenosis, were observed in the omentopexy group than in the control group. Angiography and analysis of regenerated blood vessels revealed that vessel ingrowth had started within 4 weeks and that vessel formation reached its maximal point within 6 to 12 months in the omentopexy group. In contrast, revascularization of the subepithelial region in the control group was poor even after 3 months, and vessel formation continued for as long as $\mathbf{1 2}$ months. The differences between the two groups were considered to be mainly a result of the speed of blood vessel ingrowth into the regenerated mucosa. We conclude that our prosthesis can be used safely for intrathoracic tracheal reconstruction and that omental wrapping is a useful supplementary method that reduces the occurrence of complications. (J Thorac Cardiovasc Surg 1997;113:701-11)
$\mathrm{R}$ econstruction of the trachea is now widely performed when surgery is done because of tracheal stenoses or cancers. At present, end-to-end anastomosis is considered to be the method of choice for defects up to about $6 \mathrm{~cm}$ long in adults. ${ }^{1-3}$ For patients with larger defects, several types of prostheses have been tried. However, because of the poor results obtained, they have not yet become standard surgical procedures. ${ }^{4-7}$

At present, a great deal of effort is being devoted to experimental tracheal reconstructions with the use of, for example, homograft transplantations, tracheal prostheses, and hybrid types of tracheal conduits, which use staged procedures or tissue

From the Departments of Artificial Organs ${ }^{\mathrm{a}}$ and Biomaterials, ${ }^{b}$ Research Center for Biomedical Engineering, Kyoto University, Kyoto, Japan.

Received for publication August 12, 1996; revisions requested Sept. 30, 1996; revisions received Oct. 31, 1996; accepted for publication Nov. 5, 1996.

Address for reprints: Masayoshi Teramachi, MD, Department of Artificial Organs, Research Center for Biomedical Engineering, Kyoto University, 53 Kawahara-cho, Shogoin, Sakyo-ku, Kyoto 606, Japan.

Copyright (C 1997 by Mosby-Year Book, Inc.

$0022-5223 / 97 \$ 5.00+0 \quad 12 / 1 / 79046$ engineering. ${ }^{8}$ We have been developing a new type of tracheal prosthesis that is a composite of a porous prosthesis and biocompatible collagen, and good results have already been achieved with it for reconstruction of the canine cervical trachea, ${ }^{9}$ but not for intrathoracic tracheal reconstruction in which the conditions are more severe. The aim of this study was to confirm the safety of our new prosthesis for intrathoracic tracheal reconstruction. Moreover, we evaluated the efficacy of omental wrapping of the anastomotic sites.

\section{Material and methods}

Tracheal prosthesis. The collagen-conjugated tracheal prosthesis used $(50 \mathrm{~mm}$ long with an internal diameter of 18 to $20 \mathrm{~mm}$ ) was made with a method similar to that in our previous study. ${ }^{9}$ In brief, the prosthesis comprised a cylinder of fine Marlex mesh (CR Bard, Inc., Billerica, Mass.) reinforced by 10 turns of a continuous spiral of polypropylene string ( $1 \mathrm{~mm}$ in diameter) wrapped around it. The spiral was attached to the external surface of the middle $40 \mathrm{~mm}$ of the cylinder by thermal melt-bonding at several points and further fixed with 6-0 Prolene suture (Ethicon, Inc., Somerville, N.J.) (Fig. 1). This mesh cylinder was exposed to a plasma charge to introduce peroxides onto its surface, and porcine dermal atelocollagen (supplied by Nippon Meat Packers Inc., Ibaraki, Japan) was immobilized on its surface by graft polymerization of 

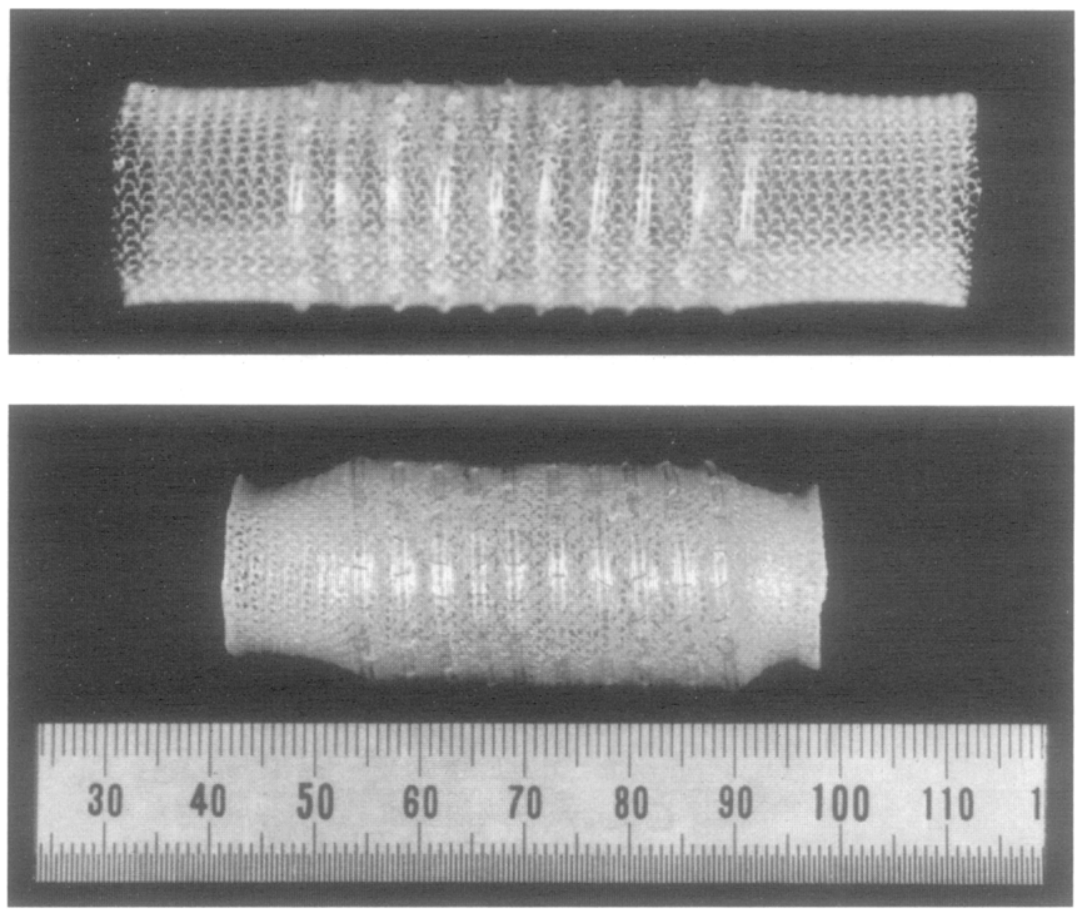

Fig. 1. Tracheal prosthesis. The mesh cylinder is reinforced with a continuous polypropylene spiral (top), then conjugated and coated with collagen (bottom).

acrylic acid. Then, to prevent air leakage from the pores of the mesh, the cylinder was coated thickly with collagen, which was finally cross-linked by exposure to ultraviolet irradiation to give it stability in vivo.

Tracheal reconstruction with or without omental wrapping. Twenty-four adult mongrel dogs, weighing 8.5 to 12 $\mathrm{kg}$, were used. Each animal underwent a right thoracotomy under general anesthesia and the intrathoracic trachea was exposed. A $45 \mathrm{~mm}$ long segment of the trachea was resected circumferentially $30 \mathrm{~mm}$ from the carina, and an endotracheal tube was placed in the distal trachea for ventilation. The proximal cut end of the trachea, including one tracheal ring, was inserted into the prosthesis and anastomosed with interrupted 3-0 Vicryl suture (Ethicon, Inc.). Then, a silicone tube $(0.7 \mathrm{~mm}$ thick, external diameter $4 \mathrm{~mm}$ less than the internal diameter of the prosthesis) was inserted into the prosthetic lumen and fixed with 4-0 PDS suture (Ethicon, Inc.). The purpose of this silicone tube was to drain any secretory products to protect the prosthesis from local infection until it was covered with host tissue. At the distal anastomosis, all the sutures were placed, then the endotracheal tube was removed from the distal trachea and all the sutures were tied quickly (Fig. 2).

Fourteen dogs also underwent laparotomy, and an omental pedicle with blood supply from the right gastroepiploic artery was prepared. The omental pedicle was placed in the right thoracic cavity through a surgically made incision in the diaphragm. The prosthesis, including both anastomotic sites, was wrapped with the omental pedicle, which was then fixed with fibrin glue and sutures (omentopexy group). In the remaining 10 dogs, air leakage from the needle holes was stopped with fibrin glue and omental wrapping was not done (control group). Thoracic drainage through a chest tube was continued after completion of the tracheal reconstruction until the animal recovered fully from anesthesia.

Bronchoscopic examination. Bronchoscopic examination was done periodically after induction of general anesthesia by an intramuscular injection of ketamine hydrochloride $(10 \mathrm{mg} / \mathrm{kg})$ and xylazine hydrochloride $(4 \mathrm{mg} / \mathrm{kg})$. The luminal surface was observed with a bronchofiberscope (model BF1T20, Olympus Optical Co., Ltd., Tokyo, Japan) to evaluate the coverage by host tissue and complications such as mesh exposure and luminal stenosis.

Histologic examination. The animals were killed with an injected overdose of sodium pentobarbital 3,6 , or 12 months after reconstruction, and en bloc resection of the prosthesis with the native trachea was done. The prosthesis was divided longitudinally, and mesh exposure on the luminal surface and luminal stenosis were evaluated macroscopically, after which longitudinal sections of the prosthesis were prepared for examination by light and scanning electron microscopy. The extent of epithelial lining on the luminal surface and the extent of inflammatory cell infiltration were evaluated in hematoxylin and eosinstained preparations by light microscopy. At least three longitudinal sections were prepared and the epithelializa- 

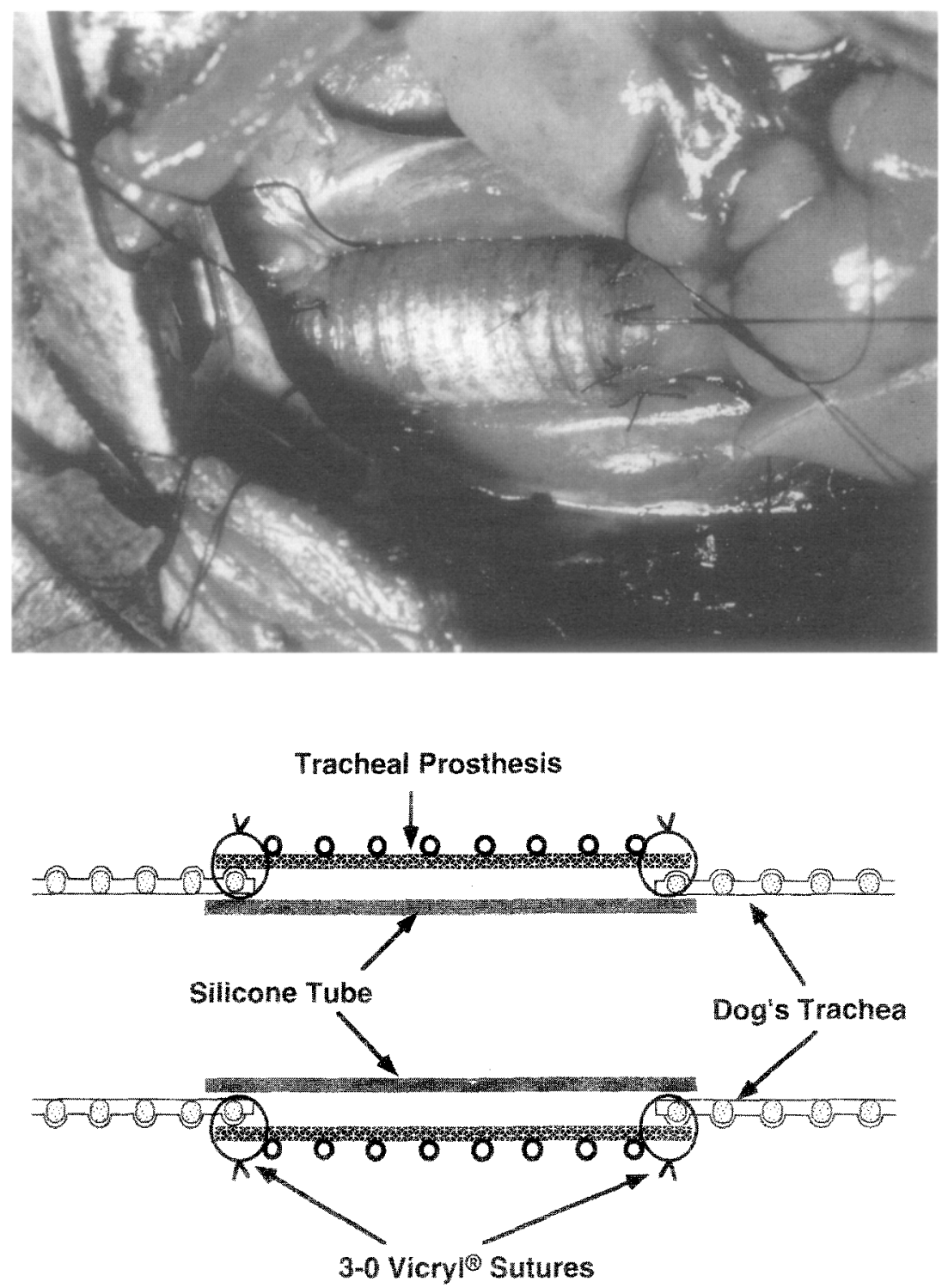

Fig. 2. A photograph taken after completion of tracheal reconstruction is shown at the top; at the bottom is a schema of the tracheal reconstruction. A silicone drainage tube is inserted into the prosthetic Iumen.

tion rate was determined with the pathologic findings. Moreover, cilia on the epithelial cells were observed by scanning electron microscope (S-450, Hitachi Ltd., Tokyo, Japan).

Comparison of the numbers of vessels in the regenerated mucosa. Each light microscopic specimen was divided into three equal parts, proximal, middle, and distal, and the numbers of vessels and capillaries in 10 rectangular areas $\left(0.66 \times 0.44 \mathrm{~mm}^{2}\right)$ of each part magnified $\times 200$ were counted. The results were expressed as means \pm standard deviations. Statistical analysis was done by Student's $t$ test, and differences at $p<0.01$ were regarded as significant.

Angiography at death. Angiography was done at the time the dogs were killed 4 weeks or 3,6 , or 12 months after tracheal prosthetic implantation in dogs in both groups and the ingrowth of new vessels into the regenerated mucosa was evaluated. Two dogs in the omentopexy group were subjected to angiography at each interval after the operation: in one dog contrast medium was injected into the celiac trunk and in the other contrast medium was injected into the right carotid artery. In the latter animal, the thoracic aorta was ligated in advance just proximal to the bifurcation of the celiac trunk to evaluate whether the blood flow through the new vessels in the regenerated mucosa originated from the omental pedicle or the bronchial arteries. One control dog was subjected to angiography at each of the same intervals and contrast medium was injected into the right carotid artery. 


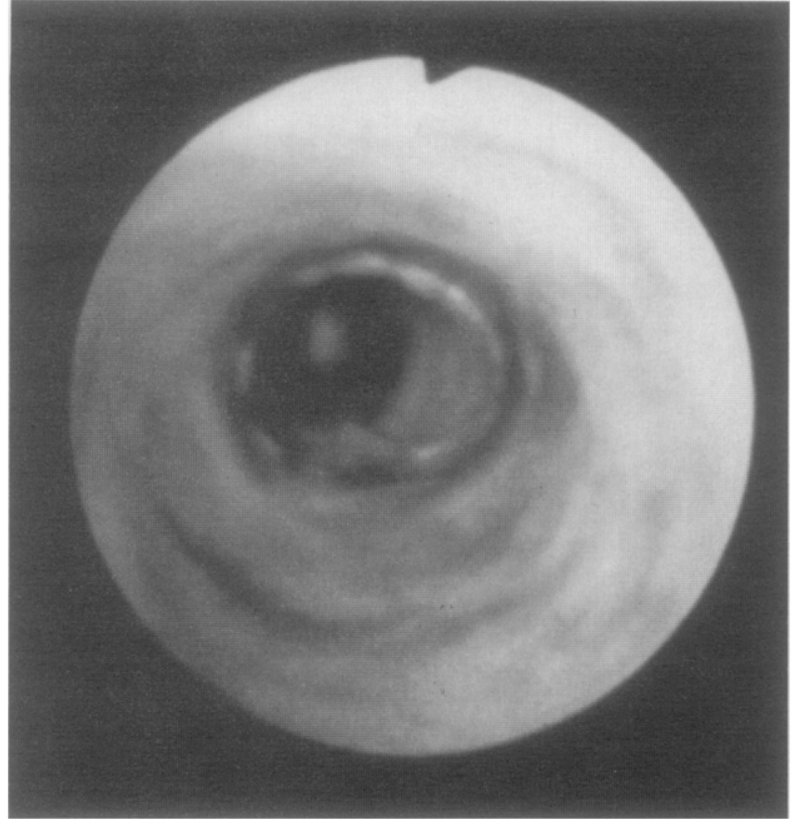

Fig. 3. Luminal surface of the prosthesis is completely covered with host tissue in a dog of the omentopexy group after 6 months.

Each animal received heparin sodium $(2 \mathrm{mg} / \mathrm{kg})$ intravenously before being killed with an overdose injection of pentobarbital. The arteries required for angiography were exposed and cannulated with an extension tube. The blood was washed out by perfusion of 1500 to $2000 \mathrm{ml}$ of physiologic saline solution containing heparin sodium (1 $\mathrm{mg} / 100 \mathrm{ml}$ ) at a pressure of $200 \mathrm{~mm} \mathrm{Hg}$ through the inserted tube. Finally, a warmed mixture of $10 \%$ wt/vol gelatin and $20 \% \mathrm{wt} / \mathrm{vol}$ barium salt $(500 \mathrm{ml})$ was injected with a syringe. This contrast medium was prepared by the method described by Inoue and colleagues. ${ }^{10}$ Immediately after this injection, ice slush was placed around the prosthesis to solidify the gelatin. The prosthesis with the native trachea was resected en bloc 20 minutes later and the surrounding tissue, including the omentum, was removed from the prosthesis and examined roentgenographically.

Animal care. Each animal received an intravenous injection of $1 \mathrm{gm}$ ampicillin on the day of the operation and a $250 \mathrm{mg} /$ day dose of ampicillin per os for 8 weeks thereafter. The silicone drainage tube placed in the prosthetic lumen was removed with the use of bronchofiberscopic forceps 4 to 8 weeks after the operation. All of the surgical procedures and killing of the animals were done in accordance with the "Guide for the Care and Use of Laboratory Animals" published by the National Institutes of Health (NIH Publication No. 85-23, revised 1985).

\section{Results}

All the animals in both groups had uneventful postoperative courses and no symptoms that necessitated killing an animal occurred. One control dog was killed at 4 weeks, two at 3 months, three at 6 months, and four at 12 months after reconstruction. In the omentopexy group, 2 dogs each were killed at 4 weeks and 3 months, four at 6 months, and five at 12 months after reconstruction, and one is still alive 18 months after reconstruction. One dog in the omentopexy group that was killed at 12 months was excluded from evaluation, because the viability of the omental pedicle appeared poor at the time of death. Overall, 13 dogs in the omentopexy group and 10 control dogs were included in the analysis to determine the efficacy of omental wrapping.

Bronchoscopic examination. Mucosal regeneration was observed in all the dogs in both groups when the silicone tube was removed. Mesh exposure was still evident at 6 months in five of the control dogs, and the regenerated mucosa was reddish even at this time. In contrast, in the omentopexy group mesh exposure was observed in only four dogs after 3 months, and within 6 months all but two had no mesh exposure and lustrous mucosa similar to that of the normal trachea (Fig. 3).

Luminal stenosis was observed in the tracheal prosthesis of four control dogs, and in three of them the stenosis was so severe that a bronchofiberscope with an external diameter of $6 \mathrm{~mm}$ could hardly be passed through (Fig. 4). In contrast, only two dogs in the omentopexy group had mild stenosis without a reddish mucosa. Although the severity of luminal stenosis in the omentopexy group increased up to 4 months, luminal stenosis continued to worsen for up to 6 to 9 months after reconstruction in the control group.

Macroscopic examination. The prostheses were incorporated into the native tracheas and there were no major complications such as erosion of the surrounding organs, abscess formation or pneumothorax, anastomotic dehiscence, or prosthetic dislocation in any of the 23 dogs that were killed. In four dogs, the prosthesis had shortened by about $5 \mathrm{~mm}$ and had bent slightly, but the animals had no symptoms.

Mesh exposure to the tracheal lumen was observed in all the control dogs and in two dogs of the omentopexy group 3 months after reconstruction. However, after 6 and 12 months, partial mesh exposure was observed in two of the eight dogs in the omentopexy group, compared with five of the seven control dogs (Table I). The areas of mesh exposure were larger in the control group than in the omentopexy group (Fig. 5). 


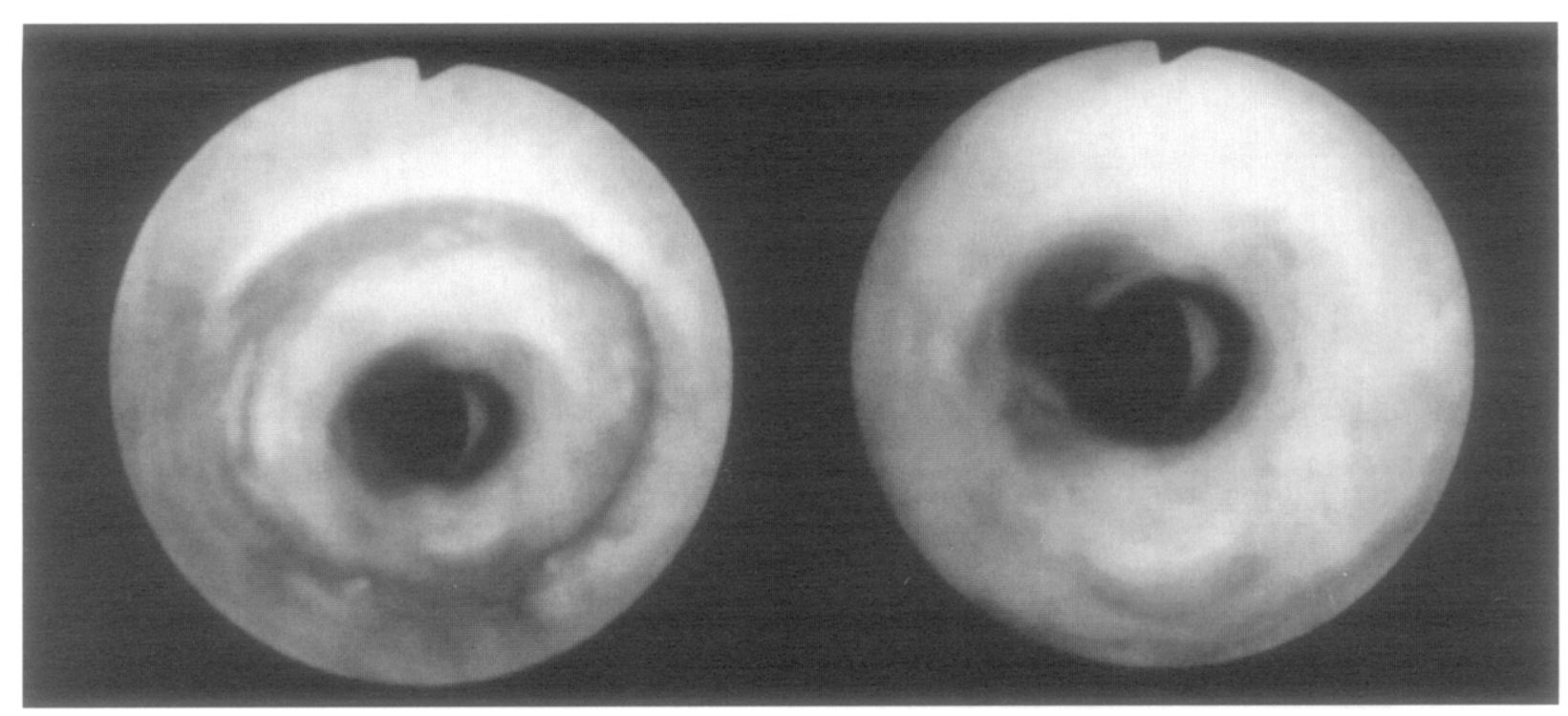

Fig. 4. Severe tracheal luminal stenosis in a control dog after 6 months. Bronchoscopic views from the proximal anastomosis (left) and just proximal to the stenosis (right).

Stenosis of the prosthetic lumen was observed in 4 of the 10 control dogs and in 2 of the 12 dogs in the omentopexy group. This stenosis was seen only in the middle of the prosthesis and not at the sites of anastomosis. The definition of stenosis we use in this paper was reduction of the tracheal luminal diameter by more than one third. Whereas both dogs in the omentopexy group had only mild stenosis, severe stenosis that reduced the diameter of the tracheal lumen by more than one half occurred in 3 of the 4 control dogs. The narrowest luminal diameter in the omentopexy group was $9 \mathrm{~mm}$ and the narrowest in the control group was $6 \mathrm{~mm}$.

Histologic examination. The dogs killed at 4 weeks were excluded from the histologic study because tissue regeneration on the prosthesis was too poor for preparation of sections for light microscopy. Therefore tissue from 10 and 9 dogs in the omentopexy and control groups, respectively, was evaluated histologically.

All the dogs in both groups had regenerated mucosa with capillary ingrowth (Fig. 6). Although the extent of epithelial lining of the regenerated mucosa on the prosthesis varied, 9 of $10 \mathrm{dogs}$ in the omentopexy group had an epithelial lining covering more than $50 \%$ of the length of the prosthesis. In contrast, after 3 and 6 months the control dogs had poor epithelial lining and after 12 months the extent of the epithelial lining was similar to that in the omentopexy group after 6 months. In each animal, ciliated columnar epithelium
Table I. Results of long-term (>6 months) observations

\begin{tabular}{|c|c|c|c|c|}
\hline $\operatorname{Dog} N o$. & $\begin{array}{c}\text { Area of } \\
\text { mesh } \\
\text { exposure }\end{array}$ & Stenosis & $\begin{array}{l}\text { Epithelialization } \\
\qquad \%)\end{array}$ & $\begin{array}{c}\text { Time after } \\
\text { implantation } \\
\text { (mo })\end{array}$ \\
\hline \multicolumn{5}{|c|}{ Omentopexy } \\
\hline 1 & Small & $17 / 20$ & 50 & 6 \\
\hline 2 & None & $10 / 19^{*}$ & 50 & 6 \\
\hline 3 & None & $13 / 17$ & 76 & 6 \\
\hline 4 & None & $15 / 17$ & 100 & 6 \\
\hline 5 & Small & $13 / 17$ & 33 & 12 \\
\hline 6 & None & $9 / 17^{*}$ & 88 & 12 \\
\hline 7 & None & $15 / 19$ & 100 & 12 \\
\hline 8 & None & $17 / 20$ & 100 & 12 \\
\hline \multicolumn{5}{|l|}{ Control } \\
\hline 1 & Large & $18 / 22$ & $<10$ & 6 \\
\hline 2 & Small & $17 / 21$ & 25 & 6 \\
\hline 3 & Large & $16 / 19$ & 32 & 6 \\
\hline 4 & Small & $11 / 18^{*}$ & 60 & 12 \\
\hline 5 & None & $7 / 18^{*}$ & 70 & 12 \\
\hline 6 & None & $6 / 17^{*}$ & 71 & 12 \\
\hline 7 & Large & $15 / 19$ & 100 & 12 \\
\hline
\end{tabular}

"Stenosis is defined as a reduction of the tracheal lumen by more than one third. The data are shown as the narrowest luminal diameter of the prosthesis/the average diameter of anastomosis sites (in millimeters).

was observed near the anastomoses, the proportion of nonciliated cuboidal epithelium increased with distance from the anastomoses, and squamous epithelium was observed in the center of the prosthesis (Fig. 7).

Scanning electron microscopy revealed that the regenerated epithelial cells near the anastomoses 

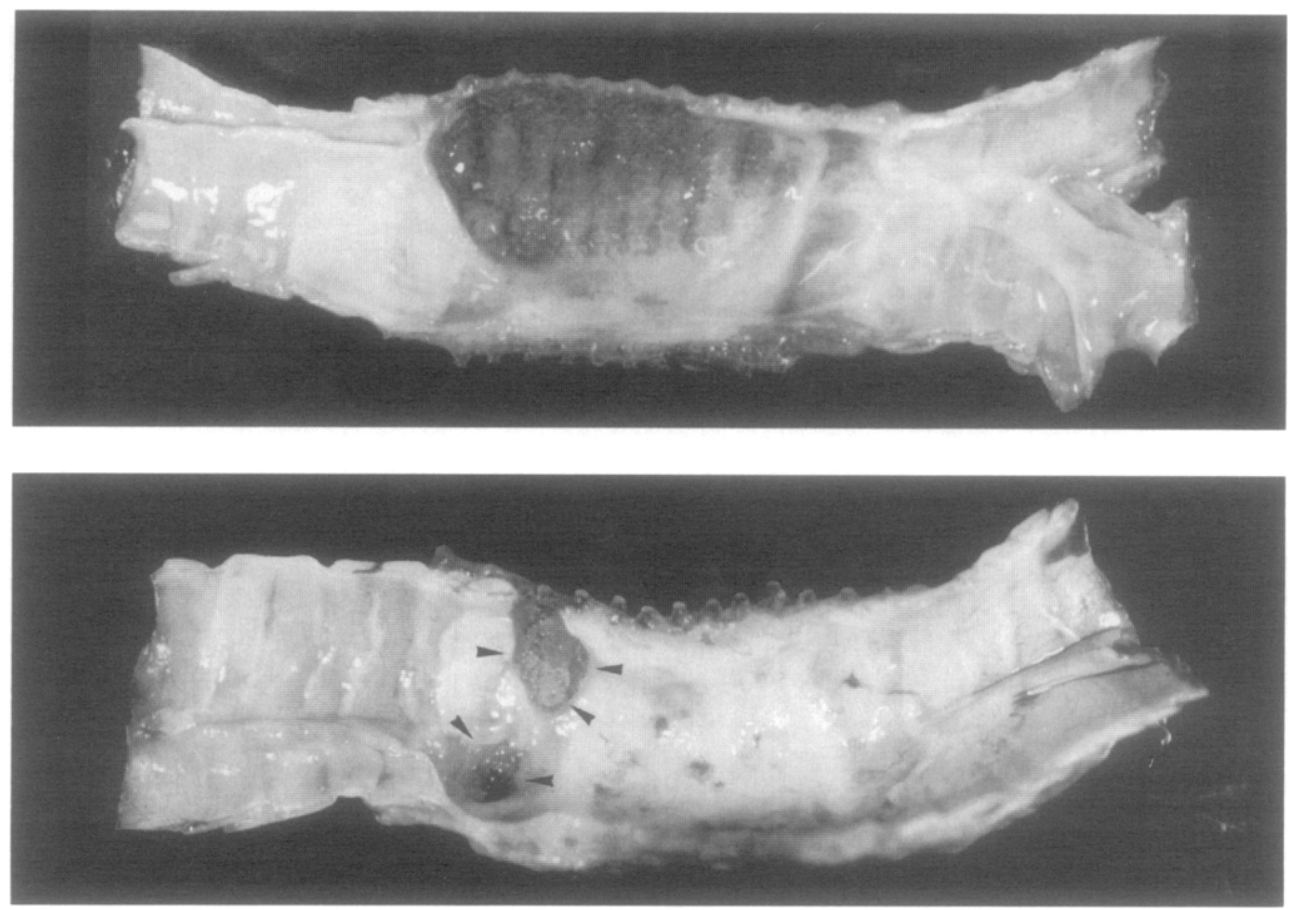

Fig. 5. Luminal surfaces of two prostheses. At the top is shown a large area of mesh exposure in a prosthesis from a control dog after 12 months. The bottom photo shows a small area of mesh exposure (arrowheads) in a prosthesis from a dog in the omentopexy group after 12 months.

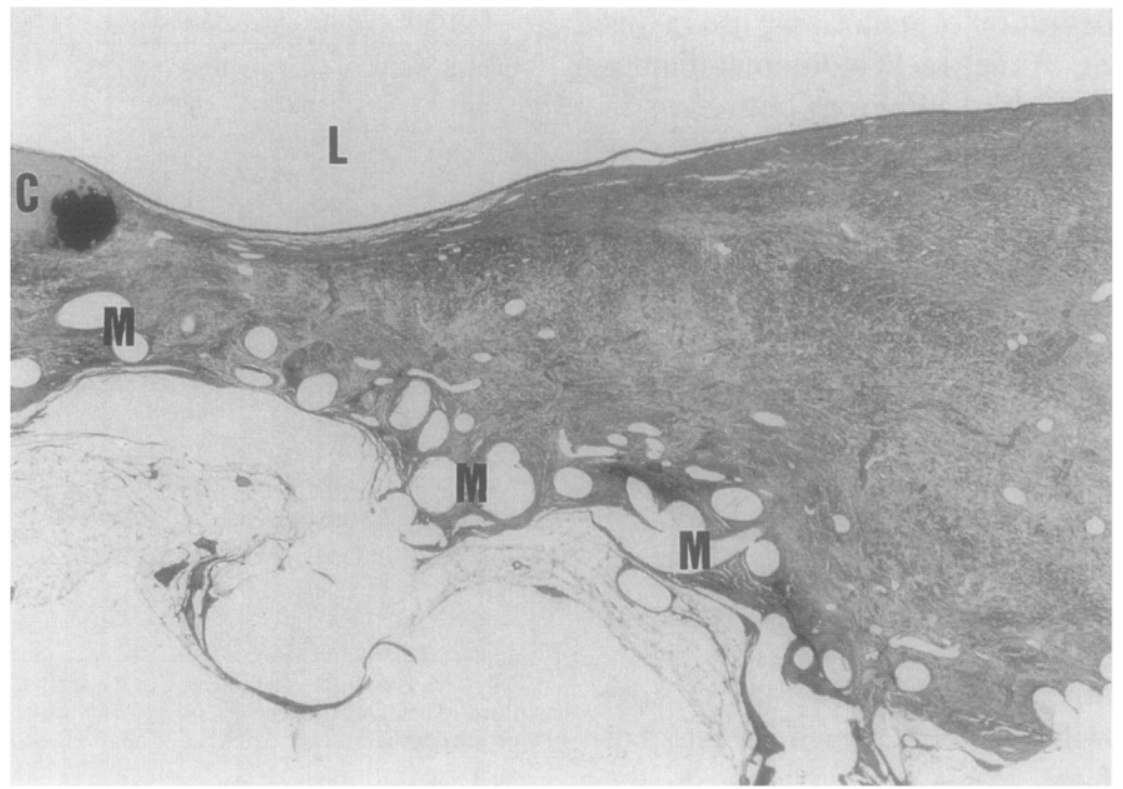

Fig. 6. Regenerated mucosa with epithelial lining on the mesh of the prosthesis from a dog in the omentopexy group after 12 months. The mucosa is thicker than the normal tracheal mucosa. $L$, Tracheal lumen; $M$, mesh of the prosthesis; $C$, tracheal cartilage (hematoxylin and eosin staining; original magnification $\times 5$ ).

possessed cilia similar to those of normal tracheal epithelium. Moreover, a few ciliated epithelial cells were observed in areas where only a squamous cell lining was demonstrated by light micros- copy (Fig. 8). Although these findings were observed in both groups, there were more ciliated cells in the omentopexy group than in the control group. 

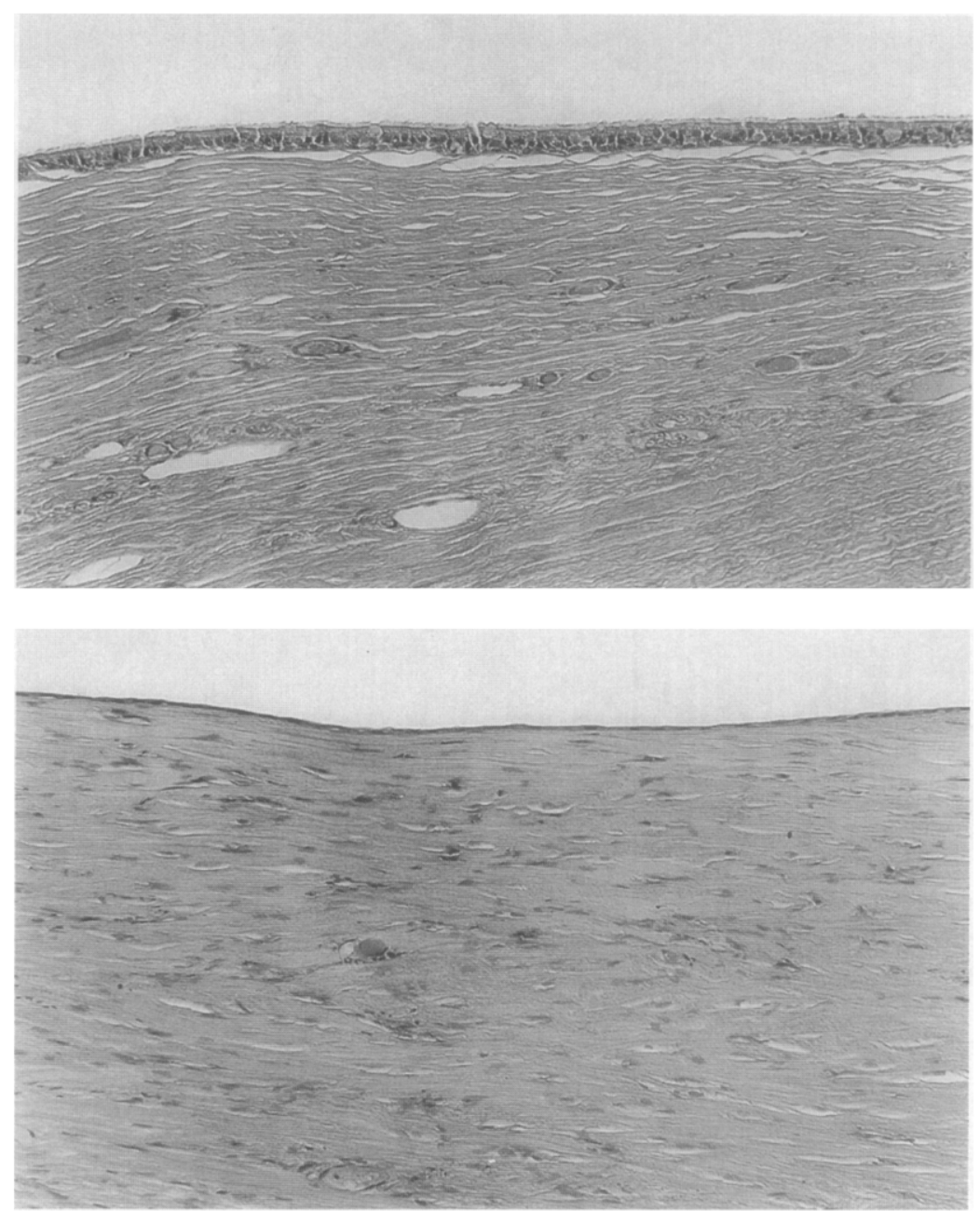

Fig. 7. Ciliated epithelium is present on the regenerated mucosa near the anastomosis (top), but only squamous epithelium is present in the middle of the prosthesis (bottom) in a dog in the omentopexy group after 12 months (hematoxylin and eosin staining; original magnification $\times 50$ ).

\section{Comparison of the numbers of vessels in the} regenerated mucosa. The numbers of vessels in the regenerated mucosa are shown in Table II. After 3 and 6 months there were significantly more vessels in the omentopexy group than in the control group. In the latter group the number of vessels increased with time after implantation, whereas in the former group the number of vessels increased from 3 to 6 months and then decreased from 6 to 12 months. There was no significant difference between the numbers of vessels in the two groups after 12 months.

Angiography at death. Angiography at the time the dogs were killed indicated that the dogs in the omentopexy group had more vessel ingrowth than the control dogs at each observation point (Fig. 9). In the control group little vessel ingrowth was observed until 6 months, and regenerated vessel ingrowth around the prosthesis was evident after 12 months. In the omentopexy group, regenerated vessel ingrowth was detected after 4 weeks only in the dog in which the contrast medium was injected into the celiac trunk. After 3 months, regenerated vessel ingrowth was observed in both dogs in which contrast medium was injected into the celiac trunk and the right carotid artery. After 6 months, it was found that some new vessels ran vertically from the outside of the prosthesis to the subepithelial region of the regenerated mucosa (Fig. 10).

\section{Discussion}

We have been developing porous types of tracheal prostheses and have already reported that our $3 \mathrm{~cm}$ 

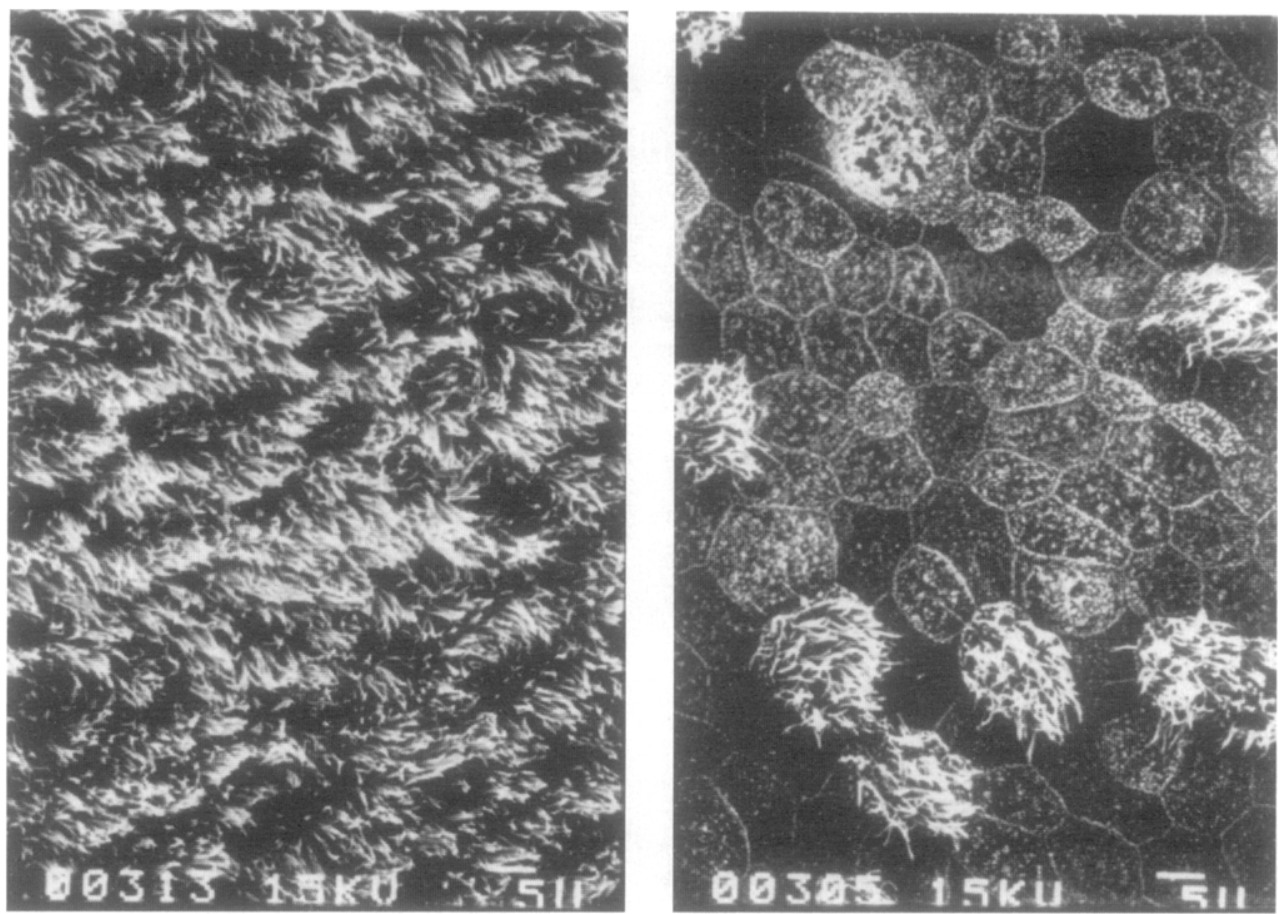

Fig. 8. Scanning electron microscopic views of the luminal surface. Dense cilia like those of normal tracheal epithelium are present on the prosthesis near the anastomosis (left) and a few ciliated epithelial cells are present in areas where only squamous cell lining was demonstrated by light microscopy (right).

Table II. Numbers of vessels in the regenerated mucosa

\begin{tabular}{llll}
\hline & \multicolumn{3}{c}{ Vessels $/ \mathrm{mm}^{2}$} \\
\cline { 2 - 4 } & \multicolumn{1}{c}{$3 \mathrm{Mo}$} & \multicolumn{1}{c}{$6 \mathrm{Mo}$} & \multicolumn{1}{c}{$12 \mathrm{Mo}$} \\
\hline Omentopexy & & & \\
$\quad$ Proximal & $45.8 \pm 15.2^{\mathrm{a} 1}$ & $56.6 \pm 21.6^{\mathrm{a} 2}$ & $34.6 \pm 12.8$ \\
$\quad$ Middle & $34.3 \pm 8.4^{\mathrm{a} 2}$ & $38.7 \pm 15.1^{\mathrm{a}}$ & $25.5 \pm 9.5$ \\
$\quad$ Distal & $69.7 \pm 34.2^{\mathrm{a} 4}$ & $80.1 \pm 27.2^{\mathrm{a} 2}$ & $47.3 \pm 13.3$ \\
Control & & & \\
$\quad$ Proximal & $31.7 \pm 16.4$ & $21.3 \pm 7.0$ & $40.6 \pm 18.4$ \\
$\quad$ Middle & $19.1 \pm 4.1$ & $26.6 \pm 9.8$ & $25.6 \pm 9.0$ \\
Distal & $37.0 \pm 12.9$ & $34.9 \pm 11.1$ & $46.2 \pm 20.5$ \\
\hline
\end{tabular}

All data are expressed as means plus or minus the standard deviation. $a_{x}$, Significantly different from the control group value $(p<0.01)$.

${ }^{a 1} p=0.008$.

$\mathrm{a}^{2} p<0.0001$

${ }^{a 3} p=0.0007$

${ }^{\mathrm{a} 4} p=0.003$.

long prosthesis has a high biocompatibility and can be used safely for cervical tracheal reconstruction. ${ }^{9}$ However, with regard to the cervical trachea, even if an end-to-end anastomosis is impossible alternative procedures such as a tracheostomy followed by secondary reconstruction with a skin flap are possible. In contrast, in patients with intrathoracic tracheal defects, such procedures are difficult or impos- sible, and consequently there are more indications for use of a tracheal prosthesis in the mediastinum than in the neck. With regard to prosthesis length, Jacobs ${ }^{11}$ referred to the fact that a relatively short ( 2 to 4 rings) circumferential tracheal replacement could be accomplished successfully even in the long term and maintained that a tracheal prosthesis should be successfully applicable for much larger defects without the need for periodic endoscopy to maintain the airway. We also considered that a $3 \mathrm{~cm}$ long prosthesis might be too short in certain cases, and therefore we designed a $5 \mathrm{~cm}$ long prosthesis and used it to reconstruct the canine cervical trachea. However, several complications such as mesh exposure and luminal stenosis occurred. ${ }^{12}$

In general, central stenosis has been one of the most frequent complications associated with porous types of tracheal prostheses. This is considered to be caused by a thick granuloma that grows in the center of the prosthesis, possibly as a result of a lack of, or delay in, formation of an epithelial lining. Therefore we believe that this can be prevented if reepithelialization of the luminal surface is completed much more quickly. Rapid tissue invasion into the pores of the mesh and rapid maturation of the subepithelial mucosa are necessary to promote epithelial lining, 

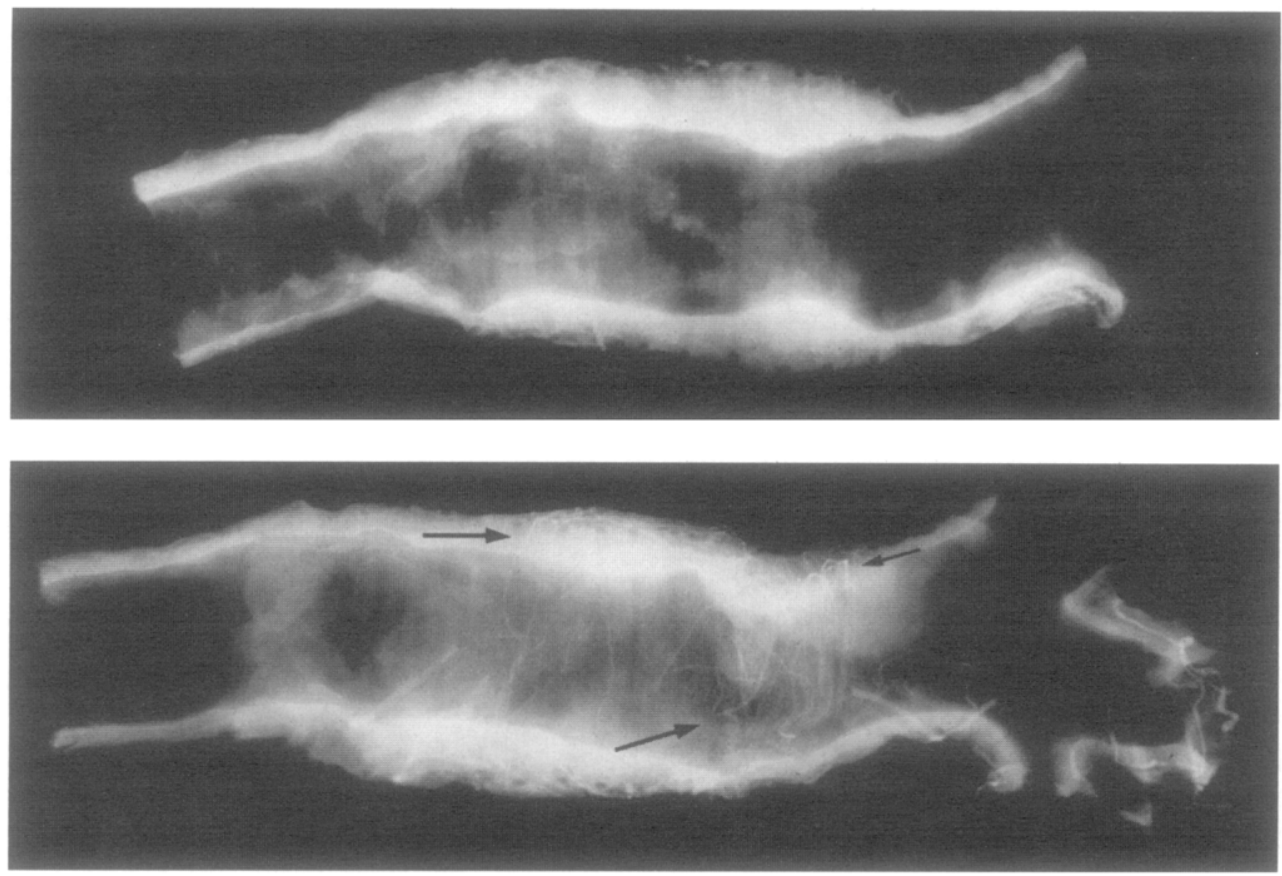

Fig. 9. Angiographic roentgenograms taken at the time the dogs were killed ( 3 months). Hardly any regenerated vessels are present in the prosthesis of a control dog (top), whereas there are a few vessels (arrows) in the prosthesis of a dog in the omentopexy group (bottom).

and there are two possible methods for fulfilling these conditions: (1) enhancement of the tissue affinity of the prosthesis itself and (2) facilitation of host cell invasion into the prosthesis. We therefore constructed a new tracheal prosthesis made of Marlex mesh and collagen sponge. The latter component was used in an attempt to improve the tissue affinity of the prosthesis. In experiments with the canine cervical trachea, this prosthesis overcame the problem of central stenosis, but mesh exposure still occurred. ${ }^{13}$

We speculate that the mesh may become exposed in places where the implanted collagen is absorbed before the host tissue has fully invaded the pores of the mesh. If this is the case, then it should be possible to avoid mesh exposure by improving the stability of the collagen. To do this, we used ultraviolet irradiation in our previous studies. However, ultraviolet irradiation alone cannot produce stronger cross-linking to collagen molecules than was achieved for this prosthesis. Chemical agents such as glutaraldehyde, which can produce stronger crosslinking than ultraviolet irradiation, do not seem suitable for tracheal prostheses because of their toxicity. ${ }^{14}$ Therefore in this study we had to abandon efforts to induce stronger cross-linking to collagen molecules and instead opted to facilitate tissue invasion into the mesh pores by omental wrapping. This approach has often been used for treatment of avascular areas, such as bronchial fistulas in respiratory operations, and it has been reported to be effective for facilitating neovascularization in tracheal autografts. ${ }^{15}$

We would like to emphasize that our prosthesis could be used safely for intrathoracic tracheal reconstruction. For intrathoracic tracheal replacement, unlike that in the neck, it is necessary to overcome more difficult conditions such as poor vascularity and negative pressure. The majority of tracheal prostheses used previously in a clinical setting were the nonporous type, probably because they overcame the problem of air leakage. However, this type of prosthesis could not solve the problem of anastomotic dehiscence and prosthesis displacement. ${ }^{4,5,7}$ Our tracheal prosthesis is porous, but the collagen-layer coating is thick enough to prevent air leakage. Deslauriers and associates ${ }^{6}$ reported that their tracheal prosthesis made of hard Marlex mesh caused massive fatal bleeding because of erosion of the innominate artery. In the light of this previous experience, therefore, our prosthesis was made of soft Marlex 


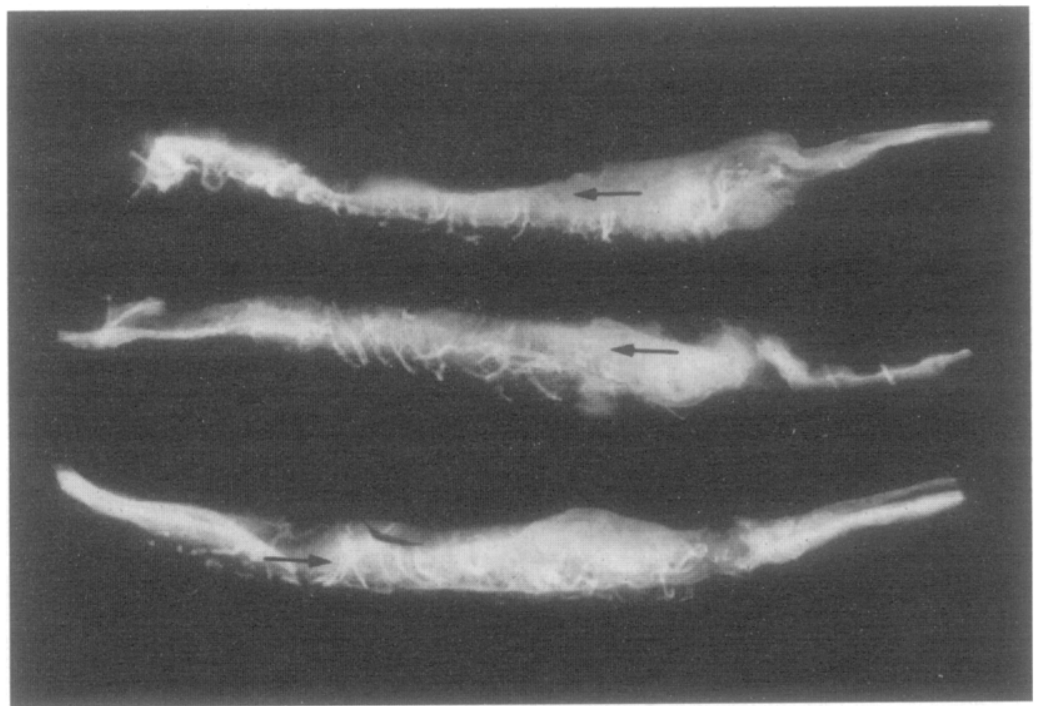

Fig. 10. Roentgenogram of longitudinal sections of the prosthesis of a dog in the omentopexy group 6 months after reconstruction. Some new vessels (arrows) run vertically from the outside of the prosthesis to the subepithelial region.

mesh reinforced with a spiral of polypropylene string, which was able to keep the tracheal lumen open against the surrounding pressure. This prosthesis has a certain degree of flexibility, which enables it to move with the neighboring trachea during respiration and as the animals move around. Therefore complications at the sites of anastomosis and erosion of the surrounding organs did not occur even during long-term follow-up for 18 months.

The results of reconstruction in the omentopexy group were apparently better than those in the control group. Mesh exposure and luminal stenosis occurred in only two dogs of the omentopexy group and, moreover, these complications were milder in degree than those in the control group. In contrast, in the control group either mesh exposure or luminal stenosis occurred in all the dogs, and the results were worse than those for cervical tracheal reconstruction. ${ }^{12}$ Therefore in conjunction with omental wrapping our prosthesis facilitated good and stable healing of the reconstructed tracheal region. Because these small areas of mesh exposure and mild stenosis did not cause any significant problems, we believe that our prosthesis could be used safely in a clinical setting with omentopexy.

In this study, we evaluated vessel ingrowth into the regenerated mucosa by counting the vessels in prepared sections and also by selective angiography at the time the dogs were killed. Omental wrapping has been used in an attempt to improve graft viability after tracheal allotransplantation for repair of circumferential defects and vessel ingrowth into the graft was reported to start within 1 week of omental wrapping. ${ }^{15,16}$ Nelson and colleagues ${ }^{17}$ reported that stable healing of tracheal prostheses was accomplished when a subepithelial network of vessels was well advanced by 6 to 8 weeks after reconstruction with omental wrapping. In our study, however, such rapid vessel ingrowth was not observed. In the omentopexy group, a vessel network was first observed after 3 months, and some vertical vessels extending from the outside of the prosthesis to the subepithelial regions were present after 6 months. In the control group, the subepithelial network of vessels was poor even after 12 months.

Although the numbers of vessels increased between 3 and 6 months after the operation and decreased thereafter in the omentopexy group, the numbers of vessels increased from 3 to 12 months in the control group. This suggests that active mucosal proliferation was complete within 6 to 12 months in the omentopexy group, but continued for at least 12 months in the control group.

The extent of the epithelial lining on the prosthesis was greater in the omentopexy group than in the control group. Good ( $>50 \%$ ) epithelial lining was observed even after 3 months in the omentopexy group, whereas in the control group this was not evident until after 12 months. These results corre- 
sponded to the changes in vessel numbers and progression of luminal stenosis observed bronchoscopically, as we have described. Accordingly, these results led us to speculate that rapid vessel ingrowth as a result of omental wrapping facilitated rapid epithelial migration from the anastomoses, the epithelial lining inhibited excessive proliferation of the regenerated mucosa, and finally the numbers of vessels, which were initially necessary to provide a good supply of blood for tissue proliferation, declined.

Neville, Bolanowski, and Soltanzadeh ${ }^{18}$ stated that the ideal tracheal prosthesis should possess six characteristics: it should (1) be airtight, (2) be of adequate consistency, (3) have good acceptance by the host, (4) cause a minimal inflammatory reaction but still be incorporated by the surrounding tissue, (5) be impervious to fibroblastic and bacterial invasion of the lumen, and (6) permit ingrowth of respiratory epithelium along the lumen. Most tracheal prostheses investigated so far have not been able to fulfill the sixth requirement. Recently, a new trial that used a tissue engineering method to induce an epithelial lining on the prosthesis before implantation was reported, ${ }^{19}$ but the tissue culture procedures involved appeared too complicated for clinical use. Almost complete epithelialization of our prosthesis, even with the $5 \mathrm{~cm}$ long version, was achieved without either staged or complex procedures, and the prosthesis possessed all the ideal characteristics outlined by Neville, Bolanowski, and Soltanzadeh. ${ }^{18}$

We conclude that our $5 \mathrm{~cm}$ long prosthesis can be used safely for intrathoracic tracheal reconstruction and that omental wrapping of the prosthesis reduces the incidence of associated complications. We found that successful reconstruction was a result of rapid vessel ingrowth into the regenerated mucosa. This prosthesis appears promising for the repair of tracheal defects in human beings.

\section{REFERENCES}

1. Grillo HC. Circumferential resection and reconstruction of the mediastinal and cervical trachea. Ann Surg 1965;162:37488.

2. Mulliken JB, Grillo HC. The limits of tracheal resection with primary anastomosis. J Thorac Cardiovasc Surg 1968;55:41821.
3. Maassen W, Greshcuchna D, Vogt-Moykopf I, Toomes H, Lfillig H. Tracheal resection: state of the art. Thorac Cardiovasc Surg 1985;33:2-5.

4. Neville WE, Bolanowski PJ, Kotia GG. Clinical experience with the silicone tracheal prosthesis. J Thorac Cardiovasc Surg 1990;99:604-13.

5. Toomes H, Mickisch G, Vogt-Moykopf I. Experiences with prosthetic reconstruction of the trachea and bifurcation. Thorax 1985;40:32-7.

6. Deslauriers J, Ginsberg RJ, Nelems JM, Pearson FG. Innominate artery rupture: a major complication of tracheal surgery. Ann Thorac Surg 1975;20:671-7.

7. Matsubara Y, Kosaba S, Ikeda S, et al. Experimental and clinical results of tracheal prosthesis. Kyobu Geka 1990;43: 368-74.

8. Grillo HC. Tracheal replacement. Ann Thorac Surg 1990;49: 864-5.

9. Okumura N, Nakamura T, Natsume T, Tomihata K, Ikada $Y$, Shimizu Y. Experimental study on a new tracheal prosthesis made from collagen-conjugated mesh. J Thorac Cardiovasc Surg 1994;108:337-45.

10. Inoue R, Nishida R, Takimoto $Y, U m S$, Hamashima $H$, Kita T. A rat esophageal varices model by partial portal vein ligation: endoscopic examination of rat esophageal varices. Jpn Arch Int Med 1992;39:125-33.

11. Jacobs JR. Investigations into tracheal prosthetic reconstruction. Laryngoscope 1988:98:1239-45.

12. Okumura $\mathrm{N}, \mathrm{Nak}$ amura $\mathrm{T}$, Takimoto $\mathrm{Y}$, et al. A new tracheal prosthesis made from collagen-grafted mesh. ASAIO J 1993; 39:M475-9.

13. Teramachi M, Kiyotani $T$, Takimoto $T$, Nakamura $T$, Shimizu Y. A new porous tracheal prosthesis sealed with collagen sponge. ASȦIO J 1995;41:M306-10.

14. Tomihata K, Burczak K, Shiraki K, Ikada Y. Cross-linking and biodegradation of native and denatured collagen. In: Shalaby SW, Ikada Y, Langer R, Williams J, editors. Polymers of biological and biomedical significance (ACS symposium series No. 540). Washington, D.C.: American Chemical Society, 1994:275-86.

15. Nakanishi R, Shirakusa T, Takachi T. Omentopexy for tracheal autografts. Ann Thorac Surg 1994;57:841-5.

16. Moriyama S, Shimizu N, Teramoto S. Experimental tracheal allotransplantation using omentopexy: histological process of rejection reaction without immunosuppression. Tohoku $\mathbf{J}$ Exp Med 1992;167:207-18.

17. Nelson RJ, Goldberg L, White RA, Shors E, Hirose FM. Neovascularity of a tracheal prosthesis/tissue complex. J Thorac Cardiovase Surg 1983;86:800-8.

18. Neville WE, Bolanowski PJP, Soltanzadeh H. Prosthetic reconstruction of the trachea and carina. J Thorac Cardiovasc Surg 1976;72:525-38.

19. Sakata J, Vacanti CA, Schloo B, Healy GB, Langer R, Vacanti JP. Tracheal composites tissue engineered from chondrocytes, tracheal epithelial cells, and synthetic degradable scaffolding. Transplant Proc 1994:26:3309-10. 